\title{
Calculation of Differential Propagation Constant Determined by Plant Morphology Using Polarimetric Measurement
}

\author{
Chufeng Hu and Nanjing Li \\ National Key Laboratory of Science and Technology on UAV, Northwestern Polytechnical University, Xian 710072, China \\ Correspondence should be addressed to Chufeng Hu; huchufeng1982@163.com
}

Received 17 April 2014; Accepted 12 June 2014; Published 29 June 2014

Academic Editor: Panayiotis Frangos

Copyright ( 92014 C. Hu and N. Li. This is an open access article distributed under the Creative Commons Attribution License, which permits unrestricted use, distribution, and reproduction in any medium, provided the original work is properly cited.

\begin{abstract}
The morphology of vegetation greatly impacts propagation of polarized electromagnetic wave. In order to validate this phenomenon, the mathematical relation between the differential propagation constant of forest vegetation and of its polarized echo is quantitatively derived by using backscattering power profile. The fluctuation of differential propagation constant with frequency is analyzed by combining the morphological characteristics of vegetation. The accurate copolarized data of 3-10 GHz frequency-domain of small trees are obtained by indoor wideband polarimetric measurement system. The results show that morphological characteristics of vegetation at different frequencies can be obtained by the differential propagation constant of polarized electromagnetic wave. At low frequencies, the plants with structural features presented oriented distribution. However, the plants show random distribution of the echoes at higher frequencies, which is mainly from the canopy. The research provides important information to choose the coherence models employed in the parameters retrieval of vegetations.
\end{abstract}

\section{Introduction}

As polarimetric radar contains more information of object spectrum characteristics, it has shown great potential in target detection and classification in remote sensing [14]. Moreover, polarimetric synthetic aperture radar (SAR) and polarimetric SAR interferometry (POLINSAR) will gain more attention and become a development trend in the future $[5,6]$. In order to use these techniques, the relation between observations and vegetation parameters (such as topography, height, and extinction coefficient) needs to be developed. Up to now, the inversion models on extracting physical parameters from radar data can be divided into three models: (1) empirical models [7], (2) electromagnetic scattering models $[8,9]$, and (3) coherence models $[10,11]$, which are well suited for POLINSAR [12-14]. However, the applications of the models are greatly influenced by such conditions when attenuation of plant is related to polarization [15]. For example, many crops, like maize, are a kind of oriented vegetation with a vertical stem; when electromagnetic wave propagates through these crops, the vertically polarized field will attenuate more rapidly than horizontally polarized one
[16]; then the orientation volume models should be employed to retrieval algorithm.

Therefore, the investigation of differential propagation constant determined by plant morphology is of great significance. In the next part, a quantitative expression between copolarized ( $\mathrm{HH}$ and VV) backscattering power profiles and differential propagation constant is derived. Then, a wideband polarimetric measurement system is constructed in a laboratory, and some small fir trees with horizontal branches and leaves are measured. In the third part, the propagation inside the vegetation is analyzed by high-resolution timedomain response, and the differential propagation constants are compared at many frequencies. The results show that the differential propagation constant of copolarization not only depends on plant morphology, but also relates to frequency.

\section{Quantitative Estimation of Differential Propagation Constant}

The propagation of wave is shown in Figure 1. After passing through space, the wave penetrates into plant from the 


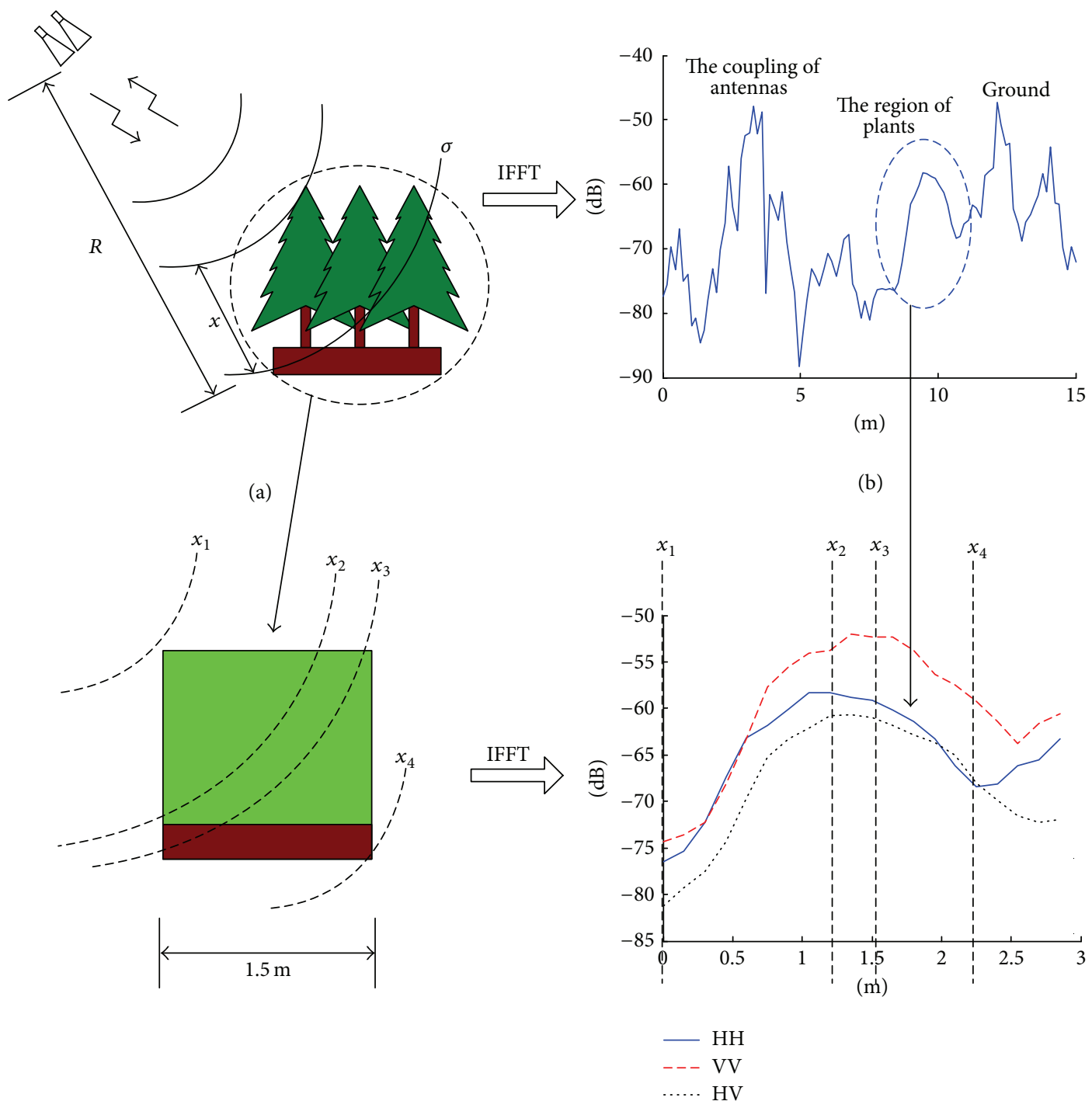

(c)

(d)

FIGURE 1: Propagation of waves in vegetation.

canopy. Due to the absorption and scattering of vegetation, the magnitude of the wave decreases. It can be expressed by an exponential factor $\exp (-k R)$, where $k$ is complex propagation constant and $R$ denotes the position along the propagation direction. If the transmitting power is $P_{0}$, then the receiving power $P$ after attenuation yields

$$
P=P_{0} \cdot e^{-k R} \text {. }
$$

If a wideband signal is transmitted and inverse Fourier transform is applied to the echo, the time-domain response of scene is obtained as shown in Figure 1(b). It shows the mutual coupling between transmit and receive antennas, the scattering of the signal by plants, and the reflection by the ground. Thus the attenuation of plants with different polarization can be analyzed by the time-domain response $[17,18]$.
Let us analyze the region of vegetation samples which can be regarded as a cube shown in Figure 1(c). The upper-left corner of the cube near the antennas is defined as the origin of coordinate; that is, $x_{1}=0$, and the energy of electromagnetic waves at different locations can be analyzed quantitatively. If the plant morphology appears at certain oriented directions, the time-domain response for different polarization channel will be different as was given in Figure 1(d).

Assume the vegetation exhibits some kinds of horizontal characteristics. When the electromagnetic waves propagate into the canopy of plants (from $x_{1}$ to $x_{2}$ ), the response of copolarization is almost the same. The influence of horizontal branches is much more obvious, so the $\mathrm{HH}$ polarization signals are weaker than the VV polarization. From the position $x_{3}$ to $x_{4}$, the $\mathrm{HH}$ polarization signals continue to be weak, and the ground begins to reflect energy. At the corner 


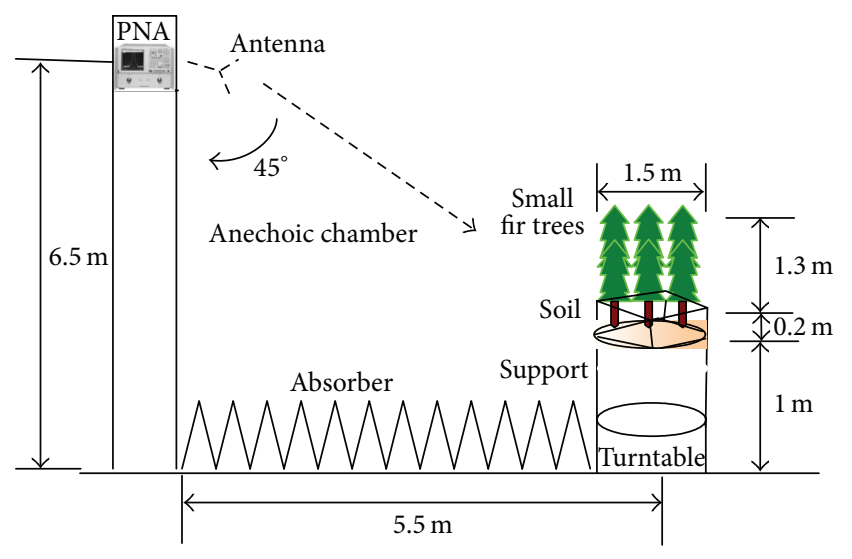

Figure 2: Measurement system.

far away from the antennas, the echoes of copolarization are approximately equal.

Considering the differential propagation constant of copolarization, the attenuation of vertical and horizontal polarizations is defined as $k_{v}$ and $k_{h}$, respectively, expressed in $\mathrm{Np} / \mathrm{m}$; the difference between them is $k_{\text {diff }}=k_{h}-k_{v}$. Assuming the transmitting power of horizontal polarization is $P_{t h}$ and the gain of the antenna is $G$, the power density $s_{t h}$ at position $x$ in the vegetation is given by

$$
s_{t h}=P_{t h} \cdot G \cdot \frac{e^{-k_{h} x}}{4 \pi R^{2}} .
$$

Let the radar cross section (RCS) at this position be equivalent to a projection area $\sigma_{h h}$; then the intercepted power $P_{i h}$ can be expressed as

$$
P_{i h}=s_{t h} \cdot \sigma_{h h}=P_{t h} \cdot G \cdot \sigma_{h h} \cdot \frac{e^{-k_{h} x}}{4 \pi R^{2}} .
$$

Since the intercepted power radiates isotropically, the returned power density $s_{r h}$ at received antenna (assuming horizontal polarization) yields

$$
s_{r h}=P_{i h} \cdot \frac{e^{-k_{h} x}}{4 \pi R^{2}}=P_{t h} \cdot G \cdot \sigma_{h h} \cdot \frac{e^{-2 k_{h} x}}{(4 \pi)^{2} R^{4}} .
$$

The received power $P_{r h}$ is obtained by multiplying returned power density and antenna effective aperture $A$. According to the antenna theory, the relation between $G$ and $A$ is as follows:

$$
A=G \cdot \frac{\lambda^{2}}{4 \pi}
$$

where $\lambda$ is the wavelength; then the received power can be written as

$$
P_{r h}=P_{t h} \cdot G^{2} \cdot \lambda^{2} \cdot \sigma_{h h} \cdot \frac{e^{-2 k_{h} x}}{(4 \pi)^{3} R^{4}}
$$

then the ratio of received and transmitted power (expressed in $\mathrm{dB}$ ) for horizontal polarization is

$$
\begin{aligned}
\mathrm{HH}(\mathrm{dB})= & 10 \log _{10}\left(\frac{P_{r h}}{P_{t h}}\right) \\
= & 10 \log _{10}\left(G^{2} \cdot \frac{\lambda^{2}}{(4 \pi)^{3} R^{4}}\right)+10 \log _{10}\left(\sigma_{h h}\right) \\
& -20 k_{h} x \cdot \log _{10} e ;
\end{aligned}
$$

similarly, the power ratio of vertical polarization is

$$
\begin{aligned}
\mathrm{VV}(\mathrm{dB})= & 10 \log _{10}\left(\frac{P_{r v}}{P_{t v}}\right) \\
= & 10 \log _{10}\left(G^{2} \cdot \frac{\lambda^{2}}{(4 \pi)^{3} R^{4}}\right)+10 \log _{10}\left(\sigma_{v v}\right) \\
& -20 k_{v} x \cdot \log _{10} e ;
\end{aligned}
$$

therefore, the difference between copolarized power ratios can be expressed as

$$
\begin{aligned}
& \mathrm{VV}(\mathrm{dB})-\mathrm{HH}(\mathrm{dB}) \\
& \quad=10 \log _{10}\left(\frac{\sigma_{v v}}{\sigma_{h h}}\right)+\left(20 \log _{10} e\right) \cdot\left(k_{h}-k_{v}\right) x .
\end{aligned}
$$

The theory and experiments prove that copolarized RCS of trees are almost equivalent [19], so the ratio of copolarized RCS can be regarded as a constant approximately, and then the differential propagation constant (expressed in $\mathrm{dB} / \mathrm{m}$ ) is

$$
k_{\text {diff }}(\mathrm{dB} / \mathrm{m})=\frac{(\mathrm{VV}(\mathrm{dB})-\mathrm{HH}(\mathrm{dB}))}{x} .
$$

Note that the derivation is also appropriated to the other two polarizations.

\section{Measurement System}

The system was constructed in the anechoic chamber of the National Key Laboratory of Science and Technology on UAV (shown in Figure 2). A vector network analyzer and four wideband horn antennas constitute a radar system that can transmit and receive vertical or horizontal signals. The antennas are aligned at an incident angle of $45^{\circ}$ with respect to ground. The distance from antennas to the center of sample is $7 \mathrm{~m}$.

The vegetation sample consists of a stand of $3 \times 3$ small fir trees, uniformly planted in a square container with side length $1.5 \mathrm{~m}$. The small fir tree is shown in Figure 3. It has a vertical stem with a diameter of about $2.5 \mathrm{~cm}$. The stem carries 5-6 horizontally oriented branches at certain distances, whose diameter is about $1 \mathrm{~cm}$. A number of needle-like leaves grown on the branches are horizontally distributed as well. The soil with $20 \mathrm{~cm}$ thickness filled in the bottom of trees.

Stepped-frequency signal [20] is generated by a vector network analyzer. The span of frequency is $2 \mathrm{GHz}$ to $12 \mathrm{GHz}$, and the interval is $10 \mathrm{MHz}$. The sample rotated by $360^{\circ}$ in 


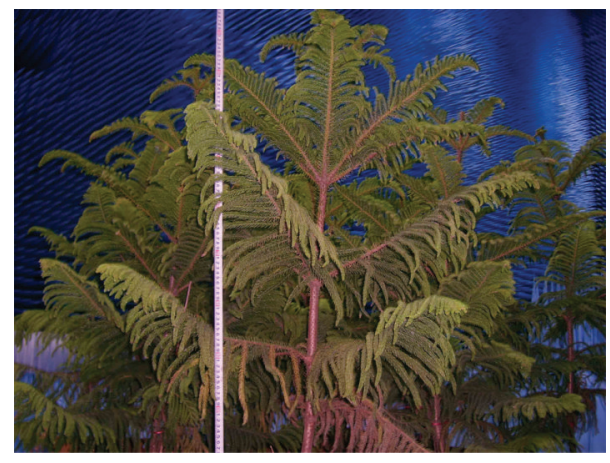

FIgURE 3: The small fir trees.

azimuth during the measurement, acquiring the polarimetric radar backscattering at 120 angles with a step of $3^{\circ}$. The echoes include many clutters, such as the mutual coupling between two antennas and residual reflections from the walls and floor as well as ceiling of the anechoic chamber. In order to remove the unwanted reflections, some pretreatments must be employed to obtain the accurate measurement data. Firstly, the plural frequency-domain data of anechoic chamber without target are subtracted from original measurement data at the same angles. Therefore, the frequency-domain response at all frequencies can be obtained as follows:

$$
y(i)=\exp \left[-j 2 \pi\left(f_{0}+i \Delta f\right) t\right],
$$

where $f_{0}$ is the start frequency, $\Delta f$ is the step of frequency, and $i=0,1, \ldots, N-1$. The phase of $y(i)$ is a linear sequence, so time-domain response can be obtained by inverse Fourier transform (IFFT) [21]:

$$
H_{l}=\frac{1}{N} \sum_{i=0}^{N-1} \exp \left[-j 2 \pi\left(f_{0}+i \Delta f\right) \times \frac{2 R}{c}\right] \times \exp \left(j \frac{2 \pi}{N} l i\right),
$$

where $R$ is the distance and $c$ is the velocity of light. The amplitude of response is gained by a modular arithmetic equation of the form shown in (13). The location of target and the other interferer resources, especially the mutual coupling between two antennas, can be seen from the high-resolution time-domain response. Consider

$$
\left|H_{l}\right|=\left|\frac{\sin \pi(l-N \Delta f \cdot 2 R / c)}{N \sin \pi(l / N-\Delta f \cdot 2 R / c)}\right|,
$$

where $l=0,1, \ldots, N-1$. The area of target can be chosen by an appropriate time-domain gating as (14); therefore, the clutters of background are eliminated effectively. Consider

$$
\left|H_{l}\right|^{\prime}= \begin{cases}\left|H_{l}\right|, & l \in \text { area of target, } \\ 0, & \text { other area. }\end{cases}
$$

The time-domain gating removes most of the unwanted signals; meanwhile, the spectrum information of original echoes is lost. If a suitable window function [22] is added to the spectrum of returning echoes, the impact caused by cutting off time-domain data will be reduced. The Hamming window is taken here as the following expression:

$$
s(n)=0.5-0.5 \cos \left(\frac{2 \pi n}{N}\right),
$$

where $n=0,1, \ldots, N-1$. Finally, a Fourier transform is applied to the time-domain response of selected region to obtain accurate frequency-domain data.

\section{Experimental Results}

The data are processed by using $1 \mathrm{GHz}$ bandwidths centered at S-band $(2-4 \mathrm{GHz}), \mathrm{C}$-band $(4-8 \mathrm{GHz})$, and $\mathrm{X}$ band $(8-12 \mathrm{GHz})$. After averaging each angle, the frequencydomain data on different polarization are transformed into time-domain response as shown in Figure 4 . The $x$-axis started in the position when waves propagate into vegetation.

From the backscattering power profiles, some obvious phenomena can be seen and the explanations are given as follows.

(1) The copolarized responses are nearly close at the beginning of propagation, and the cross polarized response is slightly lower, which can be explained by the random distribution of canopy.

(2) As the depth of penetrability increases, the echoes of $\mathrm{HH}$ polarization are decreased gradually, and the differential propagation constants begin to appear between $\mathrm{HH}$ polarization and VV polarization. The reason for this is that the horizontal branches and leaves are more and more dominated. Meanwhile, the ground begins to contribute backscattering, so the attenuation of horizontal polarization waves is greater than vertical polarization ones.

(3) At the end of penetrability, the response of vegetation tends to disappear, and the backscattering is mainly caused by ground, so each polarized echo increases gradually.

(4) All of the three bands show such phenomena and the trend is clearer at lower frequency. It indicates that the variation of differential propagation constant is related to frequency.

The phenomenon is greatly related to the morphology of vegetation. According to (10), the region of vegetation with differential polarimetric attenuation characteristic is analyzed, and the distance near the upper-left corner of cube along the propagation direction is selected at $0.5 \mathrm{~m}, 1.2 \mathrm{~m}$, and $1.5 \mathrm{~m}$, respectively. The differential propagation constants are drawn as a function of frequency given by Figure 5 . The differential propagation constants at $0.5 \mathrm{~m}$ remain substantially unchanged, indicating that the canopy has a random distribution characteristic. When the incident distance increases, the differential propagation constants at $1.2 \mathrm{~m}$ and $1.5 \mathrm{~m}$ monotonically decreased with increasing frequency, which quantitatively explains the frequency characteristics of electromagnetic wave propagation in horizontally oriented vegetation. The results explained that the propagation of polarimetric wave greatly depends on plant morphology. 


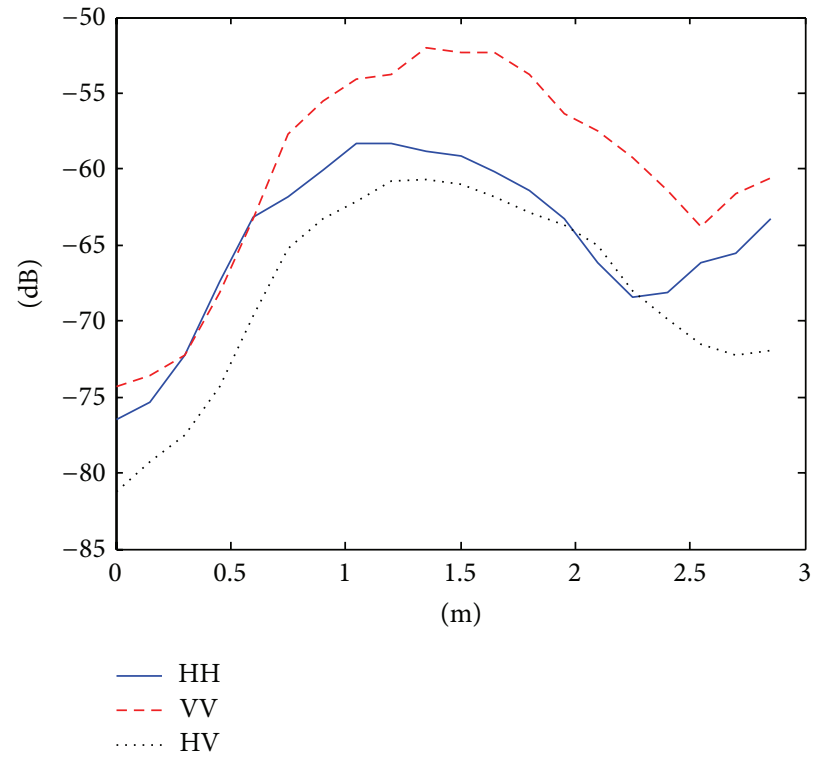

(a) S-band

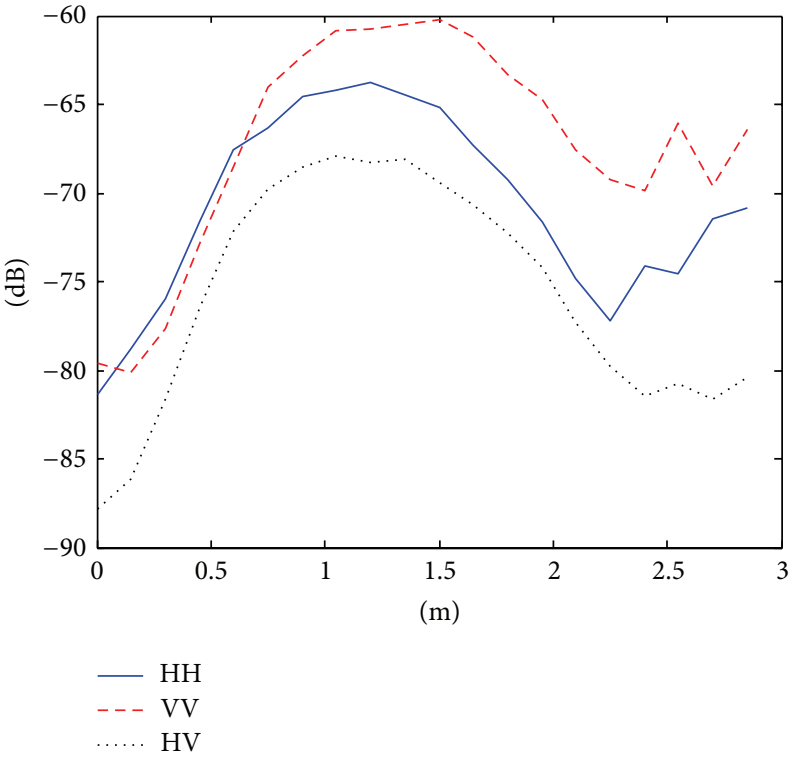

(b) C-band

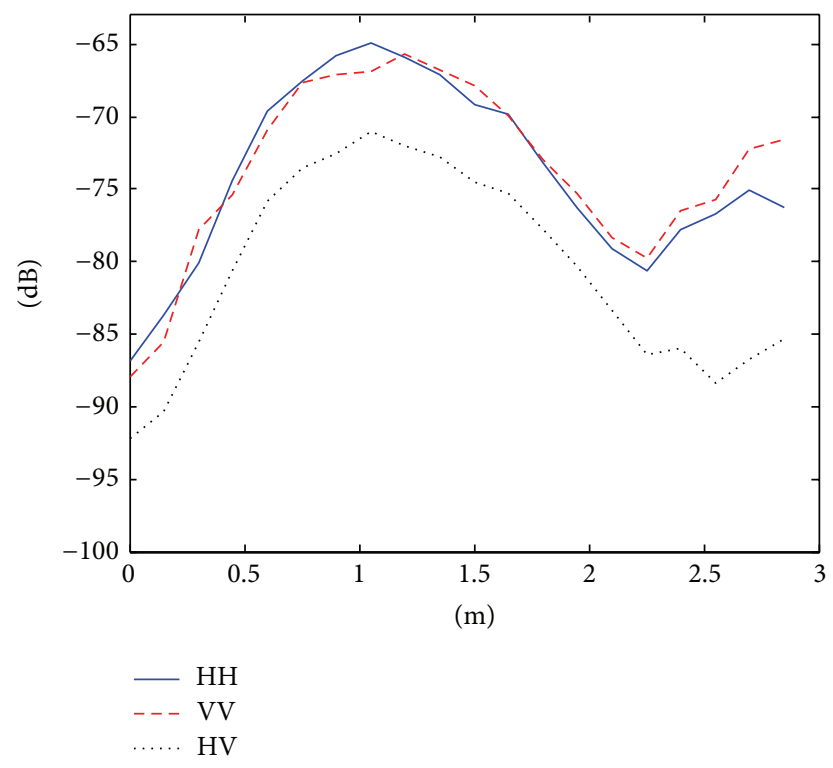

(c) X-band

FIGURE 4: Backscattering power profiles for different polarization.

\section{Conclusion}

In this paper, a sample of fir trees has been measured with a wideband polarimetric measurement system in an anechoic chamber. Backscattering power profiles reveal an expected behavior of differential propagation constants due to the horizontal orientation of vegetation. The attenuation difference is strongly determined by frequency. At low frequency, the copolarized difference is large. However, it reduces with the increases in frequency. It has been observed that the main scattering sources come from canopy at high frequency, and the impact of inner morphology can be ignored.
All of these conclusions are useful for the application of coherence models based on POLINSAR. At the same time, the qualitative scattering mechanism of oriented vegetation is investigated at different frequencies. In future research, electromagnetic wave propagation characteristics in more different morphology of vegetation will be investigated, and the effect of surface environment will be considered.

\section{Conflict of Interests}

The authors declare that there is no conflict of interests regarding the publication of this paper. 


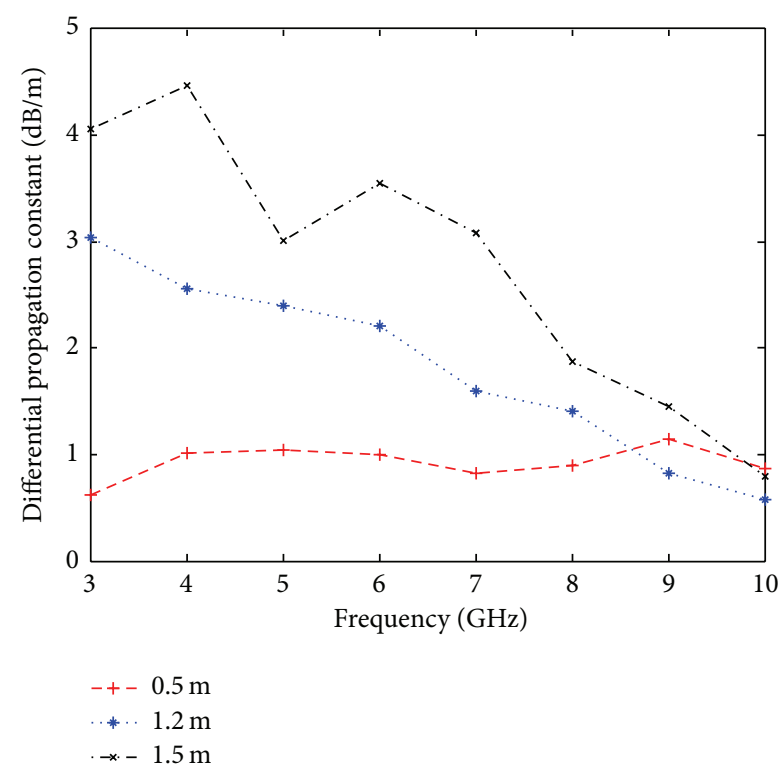

Figure 5: Differential propagation constants as a function of frequency.

\section{Acknowledgments}

The work was supported by the National Science Foundation of China (Grant nos. 61201320 and 61371023) and the Fundamental Research Funds for the Central Universities (no. 3102014JCQ01103).

\section{References}

[1] Y. Yamaguchi, "Disaster monitoring by fully polarimetric SAR data acquired with ALOS-PALSAR," Proceedings of the IEEE, vol. 100, no. 10, pp. 2851-2860, 2012.

[2] L. Coulibaly, A. Tlili, E. Hervet, and K. Adegbidi, "Mapping forest stands using RADARSAT-2 quad-polarization SAR images: a combination of polarimetric and spatial information," in Proceedings of the IEEE International Geoscience and Remote Sensing Symposium (IGARSS '12), pp. 3359-3362, Munich, Germany, July 2012.

[3] C. N. Kovama and K. Schneider, "Vegetation effects on Lband soil moisture retrieval-lessons learned from 5 years of ALOS PALSAR observations," in Proceedings of the 2012 32nd IEEE International Geoscience and Remote Sensing Symposium (IGARSS '12), pp. 1231-1234, July 2012.

[4] A. Schmitt, A. Hogg, A. Roth et al., "Shoreline classification using dual-polarized TerraSAR-X images," in Proceedings of the European Conference on Synthetic Aperture Radar, 2012.

[5] S. R. Cloude, "POLInSAR: from SIR-C to Tandem-X," in Proceedings of the IEEE International Geoscience and Remote Sensing Symposium, 2011.

[6] W. M. Boerner, "Future perspectives of SAR polarimetry with applications to multi-parameter fully polarimetric polsar remote sensing \& geophysical stress-change monitoring with implementation to agriculture, forestry \& aqua-culture plus natural disaster assessment \& monitoring within the "pacific ring of fire'”' in Proceedings of the 32nd IEEE International Geoscience and Remote Sensing Symposium (IGARSS '12), pp. 1465-1468, July 2012.

[7] F. T. Ulaby, K. Sarabandi, K. McDonald, M. Whitt, and M. C. Dobson, "Michigan microwave canopy scattering model," International Journal of Remote Sensing, vol. 11, pp. 1223-1253, 1990.

[8] Y. C. Lin and K. Sarabandi, "Electromagnetic scattering model for a tree trunk above a tilted ground plane," IEEE Transactions on Geoscience and Remote Sensing, vol. 33, no. 4, pp. 1063-1070, 1995.

[9] G. Q. Sun and K. J. Ranson, "Radar backscatter modeling of 3D vegetation structure," in Proceedings of the 1998 IEEE International Geoscience and Remote Sensing Symposium (IGARSS'98), pp. 1496-1498, Seattle, Wash, USA, July 1998.

[10] R. N. Treuhaft, S. N. Madsen, M. Moghaddam, and J. J. van Zyl, "Vegetation characteristics and underlying topography from interferometric radar," Radio Science, vol. 31, no. 6, pp. 14491485, 1996.

[11] R. N. Treuhaft and P. R. Siqueira, "Vertical structure of vegetated land surfaces from interferometric and polarimetric radar," Radio Science, vol. 35, no. 1, pp. 141-177, 2000.

[12] K. P. Papathanassiou and S. R. Cloude, "Single-baseline polarimetric SAR interferometry," IEEE Transactions on Geoscience and Remote Sensing, vol. 39, no. 11, pp. 2352-2363, 2001.

[13] S. R. Cloude and M. L. Williams, "A coherent EM scattering model for dual baseline POLInSAR," in Proceedings of the IEEE International Geoscience and Remote Sensing Symposium, vol. 3, pp. 1423-1425, Toulouse, France, July 2003.

[14] J. D. Ballester-Berman, J. M. Lopez-Sanchez, and J. FortunyGuasch, "Retrieval of biophysical parameters of agricultural crops using polarimetric SAR interferometry," IEEE Transactions on Geoscience and Remote Sensing, vol. 43, no. 4, pp. 683694, 2005.

[15] J. M. Lopez-Sanchez, J. D. Ballester-Berman, and Y. MarquezMoreno, "Model limitations and parameter-estimation methods for agricultural applications of polarimetric SAR interferometry," IEEE Transactions on Geoscience and Remote Sensing, vol. 45, no. 11, pp. 3481-3493, 2007.

[16] J. Martinez, N. Floury, T. L. Toan, A. Beaudoin, M. T. Hallikainen, and M. Mäkynen, "Measurements and modeling of vertical backscatter distribution in forest canopy," IEEE Transactions on Geoscience and Remote Sensing, vol. 38, no. 2 I, pp. $710-719,2000$

[17] K. Morrison and J. Bennett, "Time domain reflectivity imaging-a new technique," in Proceedings of the European Conference on Synthetic Aperture Radar, 2010.

[18] O. Frey and E. Meier, "Analyzing tomographic SAR data of a forest with respect to frequency, polarization, and focusing technique," IEEE Transactions on Geoscience and Remote Sensing, vol. 49, no. 10, pp. 3648-3659, 2011.

[19] R. H. Lang, R. Landry, A. Franchois, G. Nesti, and A. Sieber, "Microwave tree scattering experiment: comparison of theory and experiment," in Proceedings of the IEEE International Geoscience and Remote Sensing Symposium (IGARSS '98), pp. 2384-2386, July 1998.

[20] C. F. Hu, J. D. Xu, N. J. Li, and L. X. Zhang, "Indoor accurate RCS measurement technique on UHF band," Progress in Electromagnetics Research, vol. 81, pp. 279-289, 2008. 
[21] E. O. Brigham, The Fast Fourier Transform and Its Applications, Prentice Hall, Englewood Cliffs, NJ, USA, 1988.

[22] F. J. Harris, "On the use of windows for harmonic analysis with the discrete Fourier transform," Proceedings of the IEEE, vol. 66, no. 1, pp. 51-83, 1978. 

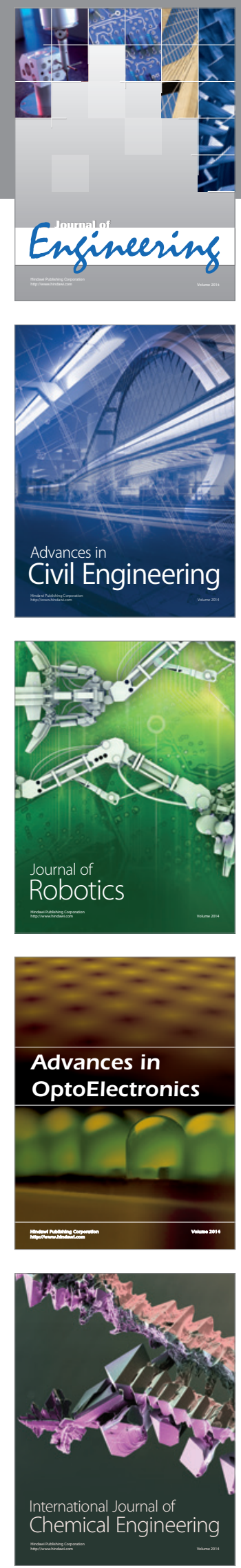

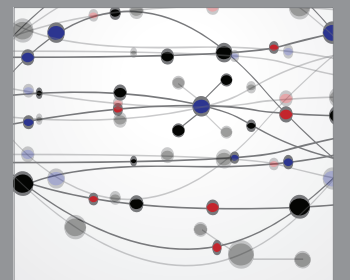

The Scientific World Journal
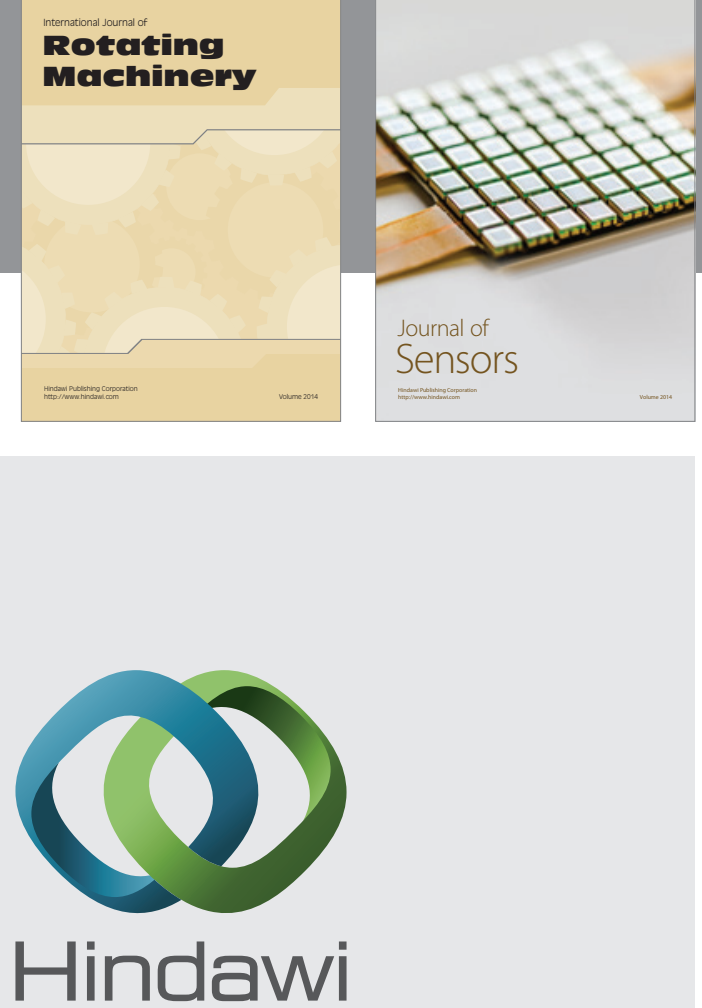

Submit your manuscripts at http://www.hindawi.com
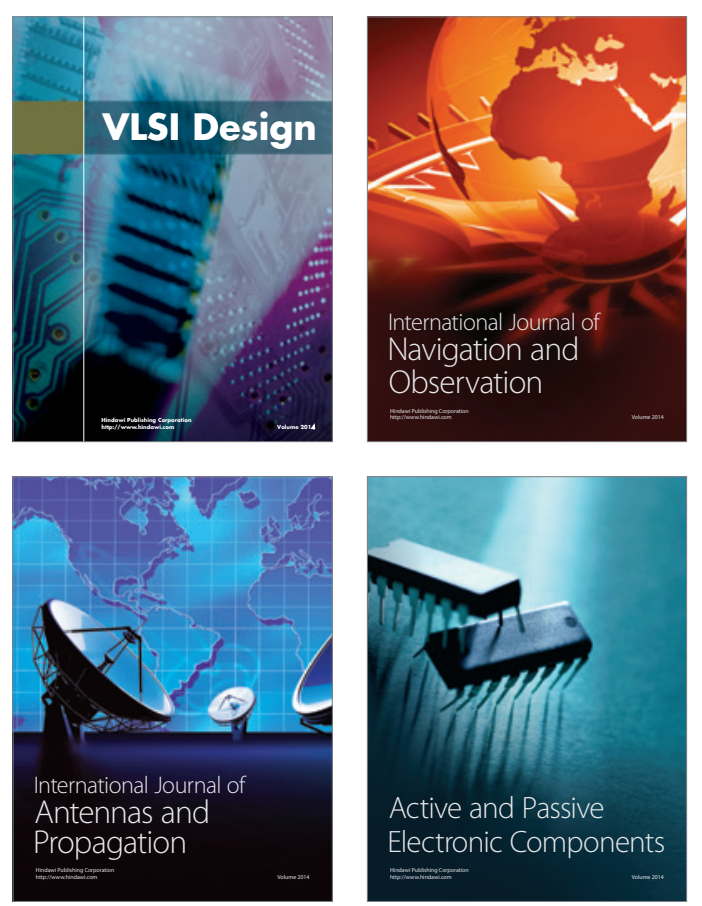
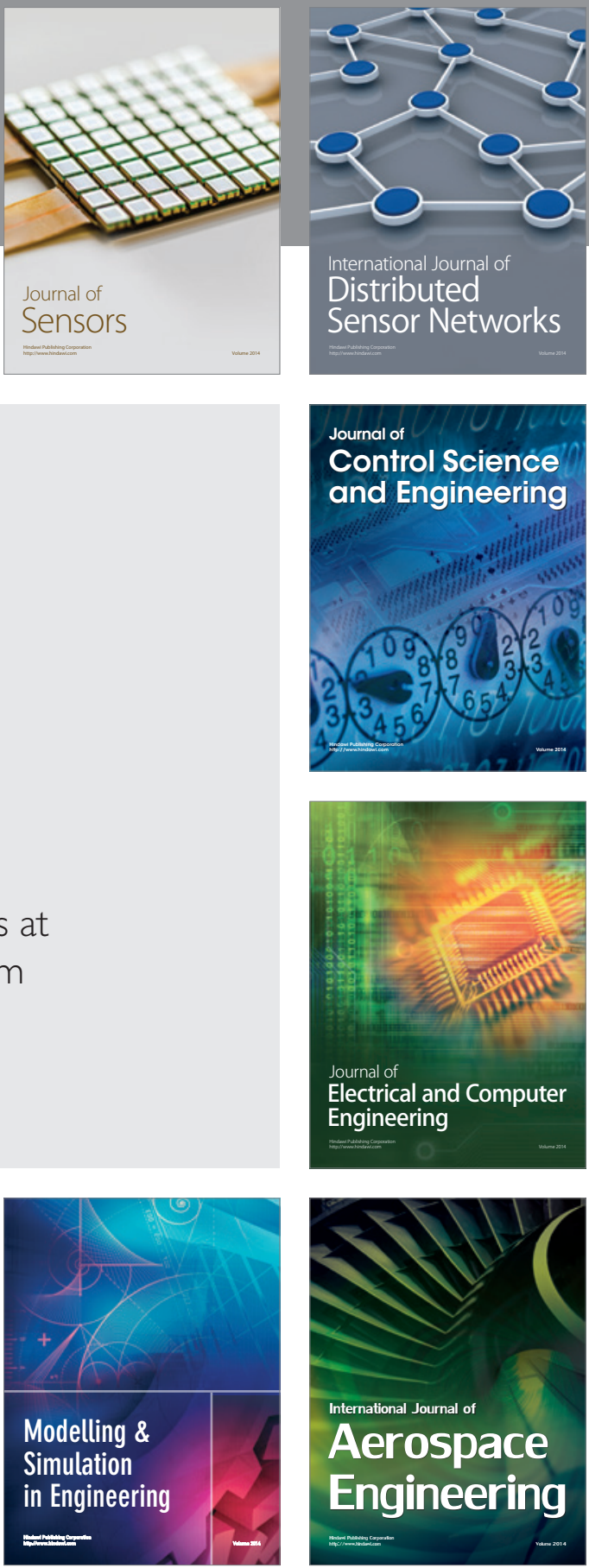

Journal of

Control Science

and Engineering
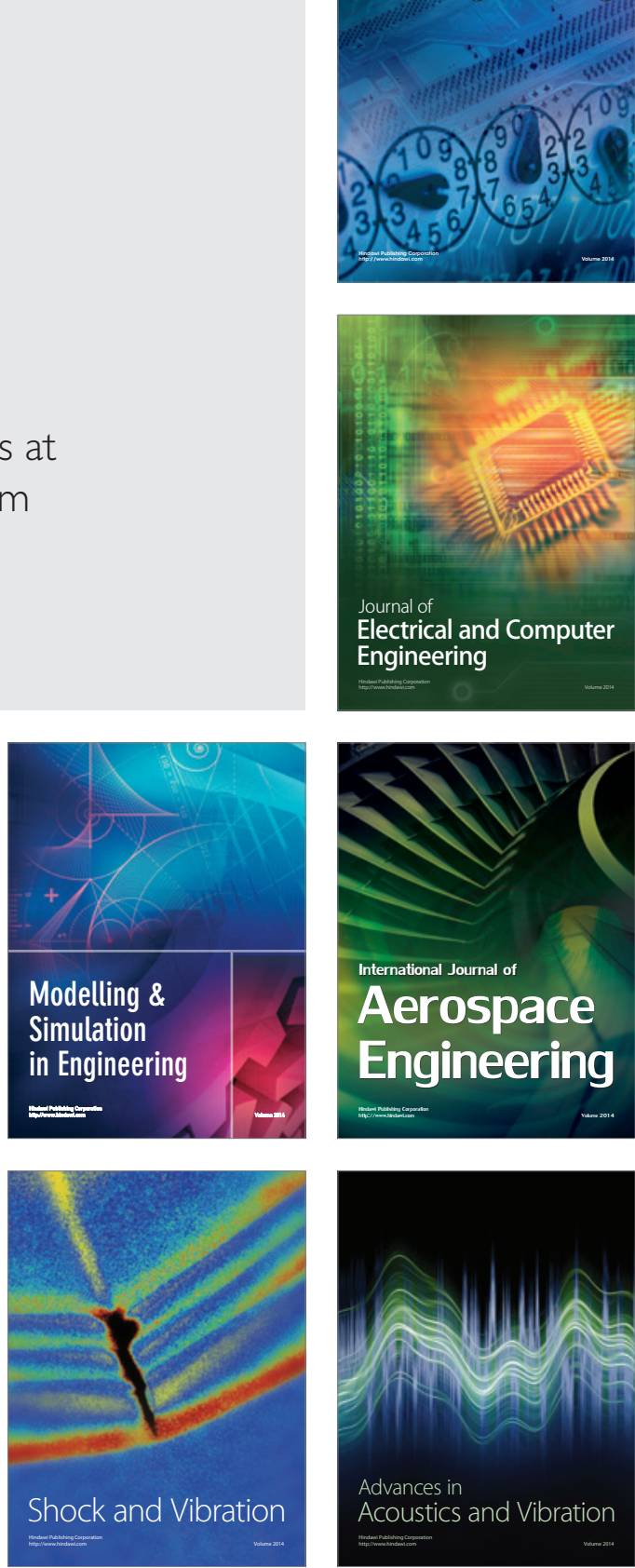